\title{
SISTEMA DE RECOMENDAÇÃO DE FERTILIZANTES E CORRETIVOS PARA A CULTURA DO ABACAXI - FERTCALC-ABACAXI ${ }^{(1)}$
}

\author{
Alexandre Paiva da Silva ${ }^{(2)}$, Víctor Hugo Alvarez V. ${ }^{(3)}$, \\ Adailson Pereira de Souza ${ }^{(4)}$, Júlio César Lima Neves ${ }^{(3)}$, \\ Roberto Ferreira Novais ${ }^{(3)} \&$ José Pires Dantas ${ }^{(5)}$
}

\begin{abstract}
RESUMO
A demanda nutricional do abacaxizeiro é alta em relação à de outras culturas e depende do cultivar, do peso do fruto, do destino da produção, do sistema e da densidade de plantio, e a variação desses fatores nem sempre está contemplada nas tabelas de adubação. O objetivo deste trabalho foi propor um Sistema de Recomendação de Fertilizantes e Corretivos para a Cultura do Abacaxi (FERTCALC-Abacaxi), elaborado a partir da sistematização e modelagem de informações de literatura e do conhecimento atual sobre adubação e nutrição mineral da cultura. O FERTCALC-Abacaxi baseia-se no método do balanço nutricional e está subdividido em dois subsistemas: o subsistema requerimento, que contempla a demanda pela planta, considerando a eficiência de recuperação dos nutrientes aplicados e uma dose para atender o critério de "sustentabilidade", e o subsistema suprimento, que compreende a oferta de nutrientes pela calagem, pelo solo, pela matéria orgânica e pelos resíduos orgânicos. Simulações realizadas com o FERTCALC-Abacaxi mostraram que o Sistema recomenda maiores doses para o cultivar Pérola em relação ao Smooth Cayenne, para plantios mais adensados e para obtenção de frutos mais pesados. Diferentemente das tabelas, as doses variaram de forma contínua com a produtividade esperada e com o teor de nutrientes no solo, sendo maiores no primeiro em relação ao segundo cultivo. Por contemplar na sua estrutura maior número de fatores envolvidos na resposta da
\end{abstract}

\footnotetext{
(1) Parte da Tese de Doutorado do primeiro autor apresentada ao Programa de Pós Graduação em Solos e Nutrição de Plantas da Universidade Federal de Viçosa - UFV. Projeto financiado pelo BNB. Recebido para publicação em março de 2008 e aprovado em maio de 2009.

(2) Professor Adjunto do Centro de Ciências e Tecnologia Agroalimentar, Universidade Federal de Campina Grande - UFCG. Rua Cel João Leite 517, CEP 58840-000 Pombal (PB). E-mail: paivadasilva@gmail.com

(3) Professor do Departamento de Solos da Universidade Federal de Viçosa - UFV. Av. PH Rolfs s/n, CEP 36570-000 Viçosa (MG). E-mails: vhav@ufv.br; julio_n2003@yahoo.com.br; rfnovais@ufv.br

(4) Professor Adjunto do Departamento de Solos e Engenharia Rural, Centro de Ciências Agrárias, UFPB. E-mail: adailson@cca.ufpb.br

(5) Professor do Centro de Ciências e Tecnologia, Universidade Estadual da Paraíba - UEPB. E-mail: gpcnpq@oi.com.br
} 
cultura à adubação, a exemplo da produtividade esperada, do cultivar, do peso do fruto, do sistema e da densidade de plantio, e considerar a contribuição dos resíduos orgânicos do cultivo anterior, o FERTCALC-Abacaxi constitui-se numa alternativa mais versátil para recomendar doses de fertilizantes e corretivos para o abacaxizeiro, embora precise ser aperfeiçoado em futuras versões e ter seu desempenho avaliado em condições práticas.

Termos de indexação: Ananas comosus L., recomendação de adubação, modelagem, balanço nutricional.

\title{
SUMMARY: FERTILIZER AND LIME RECOMMENDATION SYSTEM FOR PINEAPPLE-FERTCALC-ABACAXI
}

\begin{abstract}
The nutritional demand of pineapple plants is higher than for other crops and IT depends on cultivar, fruit weight, production destination and planting density and cultivation system. However, the variation in these factors is not always taken into account in fertilization tables. The purpose of this study was to propose a fertilizer and lime recommendation system for pineapple designated FERTCALC-Abacaxi, based on systematization and modeling of literature data and the current knowledge concerning fertilization and mineral nutrition of the crop. FERTCALC-Abacaxi is based on the nutritional balance method and is divided into two subsystems: the requirement subsystem that contemplates the plant demand, considering the efficiency of recovery of the applied nutrients and a dose to meet the requirement "sustainability"; and the supply subsystem that comprises the nutrient supply by liming, soil, organic matter, and by organic residues. Simulations with FERTCALC-Abacaxi showed that the System recommends higher doses for the Pérola cv., than for Smooth Cayenne, for denser planting systems and to obtain heavier fruits; and differently from the tables, it varied continuously with the expected productivity and soil nutrient contents, being higher in the first than in the second growing cycle. In view of the higher number of factors taken into consideration that reflect the response of the crop to fertilization, for example the expected yield, cultivar, fruit weight, planting density and system, besides taking into account the contribution of organic residues from the previous cycle, the FERTCALC-Abacaxi represents a more versatile alternative for fertilizer and lime recommendation for the pineapple crop. However, improvements in future versions of the System as well as its validation under practical conditions are necessary.
\end{abstract}

Index terms: Ananas comosus, fertilization recommendation, modelling, nutritional balance.

\section{INTRODUÇÃO}

Altas produtividades do abacaxizeiro dependem de um programa de adubação criterioso e definido conforme os objetivos da produção (Teixeira et al., 2002; Spironello et al., 2004), uma vez que a demanda nutricional da cultura é elevada e variável em função dos fatores edafoclimáticos, das práticas de manejo adotadas, do cultivar, do sistema e da densidade de plantio (Silva, 2006).

As recomendações de adubação para o abacaxizeiro nos principais Estados produtores do País fundamentam-se no conhecimento de estudiosos dessa cultura e em tabelas de adubação, em geral, despadronizadas e, em alguns casos, omissas quanto aos efeitos da produtividade esperada, do teor e da capacidade tampão (CT) de nutrientes no solo e adicionalmente do cultivar, do sistema e da densidade de plantio. Tais lacunas justificam a necessidade de uma ferramenta mais abrangente, que contemple de forma integrada a influência desses fatores, pois a adubação e a nutrição mineral afetam diretamente a produção, a qualidade dos frutos e o retorno econômico da cultura (Souza, 1999; Teixeira et al., 2002; Spironello et al., 2004; Silva, 2006).

A utilização de "Modelos" ou "Sistemas" tem se constituído numa estratégia eficiente e vantajosa para recomendar fertilizantes e corretivos (Witt et al., 1999; Haefele et al., 2003). No Brasil, essa abordagem é recente e surgiu com o desenvolvimento do Sistema de Recomendação de Adubação para Eucalipto NUTRICALC (Barros et al., 1995), posteriormente aperfeiçoado e utilizado para diferentes grupos de culturas (Tomé Junior \& Novais, 2000; Oliveira et al., 2005; Silva, 2006).

Sob a designação atual de FERTCALC, os Sistemas de Cálculo e de Recomendação de Fertilizantes e Corretivos têm se mostrado mais criteriosos, pois 
contemplam, de forma integrada, maior número de fatores envolvidos na resposta da cultura às doses (Oliveira et al., 2005). Além disso, apresentam base científica consistente, permitindo recomendações mais abrangentes e passíveis de contínuo aperfeiçoamento (Santos et al., 2008). No entanto, a qualidade das recomendações depende de informações sobre dinâmica e interação dos nutrientes com o solo e da eficiência de absorção e de utilização pelas plantas, as quais influenciam diretamente a parametrização do modelo (Cantarutti et al., 2007).

Este trabalho teve por objetivo propor uma ferramenta alternativa para recomendação de adubação do abacaxizeiro, denominado Sistema de Recomendação de Fertilizantes e Corretivos para a Cultura do Abacaxizeiro (FERTCALC-Abacaxi), elaborado por meio da sistematização e modelagem de informações disponíveis na literatura sobre adubação e nutrição mineral da cultura.

\section{MATERIAL E MÉTODOS}

\section{Desenvolvimento do sistema}

O FERTCALC- Abacaxi foi estruturado com base nas proposições de Oliveira et al. (2005), combinando modelos mecanísticos e empíricos de modo a utilizar a menor quantidade de informações para alimentar o Sistema, sem, contudo, comprometer a exatidão das recomendações. Além disso, o Sistema foi estruturado de modo a permitir sua constante atualização e, ou, o desenvolvimento de novas versões à medida que novas informações e dados forem apresentados na literatura ou gerados pelos usuários.

As recomendações feitas pelo FERTCALC-Abacaxi estão baseadas no princípio do balanço nutricional e resultam da diferença entre o requerimento de nutrientes pela cultura para alcançar determinada produtividade e seu suprimento pelo solo e pelos resíduos orgânicos, considerando o critério de sustentabilidade.

O FERTCALC-Abacaxi está subdividido em dois subsistemas: (a) o subsistema requerimento (REQ), que contempla a demanda pela planta, considerando a eficiência de recuperação dos nutrientes aplicados e uma dose para atender o critério de "sustentabilidade"; e (b) o subsistema suprimento (SUP), que compreende a oferta de nutrientes pela calagem, pelo solo, pela matéria orgânica e pelos resíduos orgânicos.

\section{Subsistema requerimento}

Para determinar o requerimento de nutrientes pelo abacaxizeiro (Figura 1), define-se inicialmente a produtividade esperada, denominada no Sistema de Produtividade a ser Efetivamente Alcançada (PEF), a qual considera as variações dos seguintes fatores: cultivar, peso do fruto, sistema e densidade de plantio e nível tecnológico do sistema de produção. Os valores de PEF são definidos a partir dos dados apresentados no quadro 1, utilizando-se as seguintes expressões:

a) Para o sistema de plantio em fileiras simples

$$
\mathrm{PEF}=\mathrm{PM}_{\mathrm{e}} \mathrm{F} \times 10 /(\mathrm{a} \mathrm{x} \mathrm{b}) \times \alpha \times \beta
$$

b) Para o sistema de plantio em fileiras duplas

$$
\mathrm{PEF}=\mathrm{PM}_{\mathrm{e}} \mathrm{F} \times\{10 / \mathrm{c}[(\mathrm{d}+\mathrm{e}) / 2]\} \times \alpha \times \beta(2)
$$

em que PEF: produtividade a ser efetivamente alcançada, em tha- ${ }^{-1} \mathrm{PM}_{\mathrm{e}} \mathrm{F}$ : peso médio do fruto a ser produzido, em kg; a: espaçamento entre as fileiras simples, em m; b: espaçamento entre as plantas dentro das fileiras simples, em m; c: espaçamento entre as fileiras duplas, em m; d: espaçamento entre as fileiras simples dentro das fileiras duplas, em m; e: espaçamento entre as plantas dentro das fileiras simples, em m; $\alpha$ : fator de correção de densidade ${ }^{(5)}$, variável em função do nível tecnológico adotado, sendo 0,7 para sistemas de produção com baixo nível tecnológico, 0,8 para sistemas com nível tecnológico médio, e 0,9 para sistemas com alto nível tecnológico; $\beta$ : fator de correção para classe de fruto a ser produzido $^{(6)}, 1,3$

Estabelecido o valor da PEF, o Sistema estima, a partir de equações de regressão, a quantidade de matéria seca total que será produzida (Quadro 2). Em seguida, o Sistema determina as quantidades de matéria seca acumuladas nas diferentes partes da planta, multiplicando a quantidade de matéria seca total produzida pelo percentual de matéria seca alocado em cada parte, considerando a distribuição percentual em função dos cultivares: Pérola - raízes e pedúnculo

\footnotetext{
(5) $\mathrm{O}$ fator de correção de densidade resulta da constatação de que o stand final é sempre inferior à densidade teórica, havendo em função do grau de tecnificação do cultivo perdas entre 10 e $30 \%$. O nível tecnológico adotado no FERTCALC-Abacaxi engloba as variações quanto aos seguintes aspectos: acesso a crédito e assistência técnica, mecanização do cultivo e colheita, e forma de comercialização da produção. São considerados com nível tecnológico alto $(0,9)$ sistemas em que os produtores possuem capital próprio, apresentam cultivo mecanizado, recebem orientação técnica e comercializam a produção diretamente sem intermediários. Para sistemas de produção cujo acesso de crédito é financiado, e que apresentam o cultivo e a colheita sem mecanização, sem assistência técnica e cuja comercialização é feita por meio de intermediários, adota-se o fator de correção 0,7. As combinações diferentes das apresentadas são incluídas no nível tecnológico médio e, portanto, correspodem ao fator de nível tecnológico 0,8 .

${ }^{(6)}$ A heterogeneidade no peso dos frutos ocorre devido à influência dos fatores edafoclimáticos e de manejo, especialmente no que concerne à adubação, nutrição mineral, indução floral e tamanho das mudas. Assim, haverá por ocasião da colheita frutos com peso superior e inferior aos da categoria escolhida, razão pela qual o Sistema considera que apenas $70 \%$ dos frutos da produtividade efetiva enquadram-se na classe de peso definida inicialmente.
} 


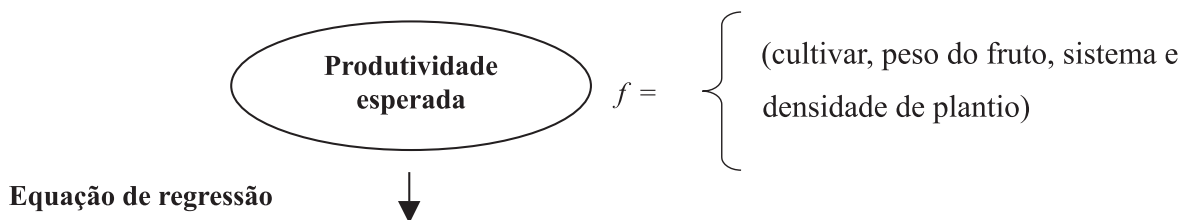

Quantidade de matéria seca (MS) produzida

$\times$ Percentual de matéria seca alocado em cada parte da planta

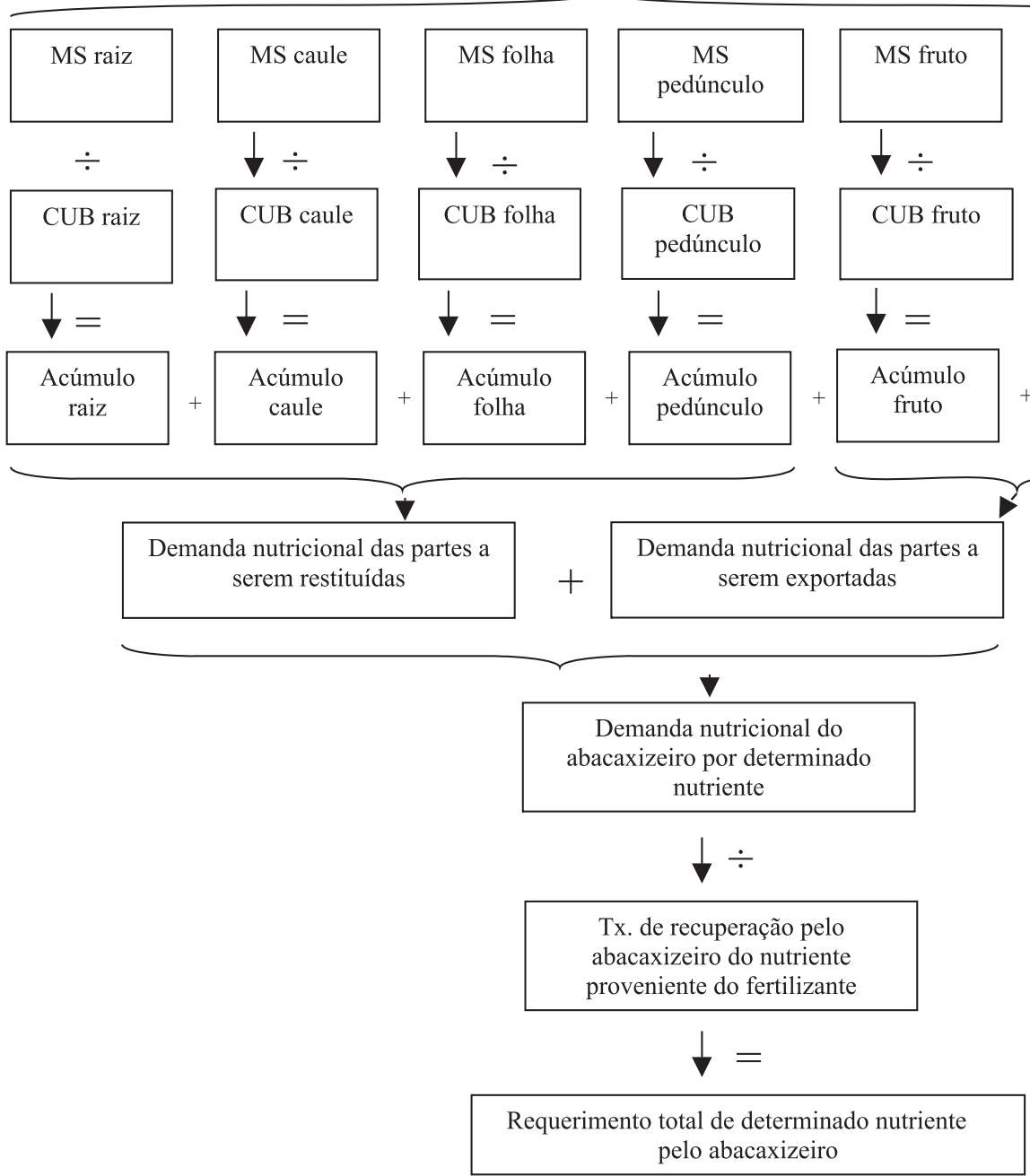

Figura 1. Fluxograma genérico utilizado pelo FERTCALC-Abacaxi para estimar o requerimento de nutrientes pelo abacaxizeiro.

(4,0\%); caule (18,0\%); folhas $(68,0 \%)$; frutos $(15 \%)$ e mudas $(6,0 \%)$; Smooth Cayenne - raízes $(6,0 \%)$; caule $(22,0 \%)$; pedúnculo $(3,0 \%)$; folhas $(64,0 \%)$; frutos $(15 \%)$ e mudas $(5,0 \%)$.

O passo seguinte consiste em determinar o acúmulo de nutrientes nas diferentes partes da planta, o que pode ser obtido dividindo-se as quantidades de matéria seca acumulada nas diferentes partes pelos respectivos coeficientes de utilização biológica (CUB) de cada nutriente.
Os valores de CUB são expressos em kg kg-1 e indicam a produção de matéria seca por unidade de nutriente acumulada, representando na prática uma medida da eficiência da planta em converter nutriente absorvido em matéria seca (Barros et al., 1995). Os valores de CUB são influenciados por fatores edafoclimáticos, biológicos e de manejo, podendo-se afirmar, em geral, que, para uma mesma produtividade, plantas com valor de CUB mais alto de determinado nutriente são mais eficientes do que 
Quadro 1. Classes de peso de frutos e espaçamentos utilizados, para os cultivares Pérola e Smooth Cayenne, no sistema de fileiras simples e duplas

\begin{tabular}{|c|c|c|c|c|c|}
\hline \multirow[b]{2}{*}{ Cultivar } & \multirow[b]{2}{*}{ Classe de peso ${ }^{(1)}$} & \multirow[b]{2}{*}{ Simples } & \multicolumn{3}{|c|}{ Espaçamento/densidade } \\
\hline & & & Densidade $^{(2)}$ & Dupla & Densidade $^{(3)}$ \\
\hline & & $\mathrm{m}$ & plantas ha ${ }^{-1}$ & $\mathrm{~m}$ & plantas ha ${ }^{-1}$ \\
\hline \multirow[t]{4}{*}{ Pérola } & I $\quad(0,9-1,2 \mathrm{~kg})$ & $0,80^{(4)} \times 0,30^{(5)}$ & 41.666 & $0,90^{(6)} \times 0,40^{(7)} \times 0,35^{(8)}$ & 43.960 \\
\hline & II $(1,2-1,5 \mathrm{~kg})$ & $0,80 \times 0,40$ & 31.250 & $0,90 \times 0,40 \times 0,40$ & 38.460 \\
\hline & III $(1,5-1,8 \mathrm{~kg})$ & $0,90 \times 0,30$ & 37.040 & $1,00 \times 0,40 \times 0,40$ & 35.710 \\
\hline & IV $(1,8-2,1 \mathrm{~kg})$ & $0,90 \times 0,35$ & 31.700 & $1,20 \times 0,40 \times 0,30$ & 41.670 \\
\hline Smooth & I $\quad(0,9-1,2 \mathrm{~kg})$ & $0,90 \times 0,40$ & 27.780 & $1,20 \times 0,40 \times 0,40$ & 31.250 \\
\hline \multirow{5}{*}{ Cayenne } & II $(1,2-1,5 \mathrm{~kg})$ & $0,80 \times 0,30$ & 41.666 & $0,90 \times 0,40 \times 0,35$ & 43.960 \\
\hline & III $(1,5-1,8$ kg) & $0,80 \times 0,40$ & 31.250 & $0,90 \times 0,40 \times 0,40$ & 38.460 \\
\hline & IV $(1,8-2,1 \mathrm{~kg})$ & $0,90 \times 0,30$ & 37.040 & $1,00 \times 0,40 \times 0,40$ & 35.710 \\
\hline & $\mathrm{V}(2,1-2,4 \mathrm{~kg})$ & $0,90 \times 0,35$ & 31.700 & $1,20 \times 0,40 \times 0,30$ & 41.670 \\
\hline & VI $\quad(>2,4 \mathrm{~kg})$ & $0,90 \times 0,40$ & 27.780 & $1,20 \times 0,40 \times 0,40$ & 31.250 \\
\hline
\end{tabular}

(1) Adaptado de Almeida et al. (2005) e Silva (2006). ${ }^{(2)}$ Densidade teórica calculada para o sistema de plantio em fileiras simples pela expressão 10.000 / ( $\mathrm{a}$ b), em que a: espaçamento entre fileiras, em m; e b: espaçamento entre plantas dentro das fileiras simples, em m. ${ }^{(3)}$ Densidade teórica calculada para o sistema em fileiras duplas pela expressão 10.000/e [(c + d)/2], em que c: espaçamento entre as fileiras duplas, em m; d: espaçamento entre as plantas dentro das fileiras simples; e: espaçamento entre fileiras simples dentro da fileira dupla, em m.

Quadro 2. Equações de regressão para estimar a produção de matéria seca dos abacaxizeiros $(\hat{y})$ Pérola e Smooth Cayenne, em função da produtividade esperada (x)

\begin{tabular}{|c|c|c|}
\hline Equação & $\mathbf{R}^{2}$ & $\mathbf{n}$ \\
\hline 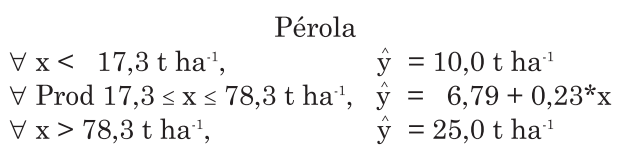 & 0,71 & 15 \\
\hline \begin{tabular}{ll}
\multicolumn{2}{c}{ Smooth Cayenne } \\
$\forall \mathrm{x}<36,0 \mathrm{tha}^{-1}$, & $\hat{\mathrm{y}}=12,0 \mathrm{tha}^{-1}$ \\
$\forall \operatorname{Prod} 36,0 \leq \mathrm{x} \leq 89,0 \mathrm{tha}^{-1}$, & $\hat{\mathrm{y}}=5,72+0,18^{*} \mathrm{x}$ \\
$\forall \mathrm{x}>89,0 \mathrm{tha}^{-1}$, & $\hat{\mathrm{y}}=22,0 \mathrm{tha}^{-1}$
\end{tabular} & 0,85 & 16 \\
\hline
\end{tabular}

Fonte: Adaptado de Silva (2006). *: significativo a 5 \%; n: número de dados utilizados para ajustar as equações.

plantas com valor de CUB mais baixo (Oliveira et al., 2005; Santos et al., 2008). Por falta de informações na literatura que permitissem relacionar os valores de CUB com os fatores que influenciam essa variável, foram adotados valores médios (Quadro 3) na primeira versão do FERTCALC-Abacaxi.

O somatório das quantidades de nutrientes acumuladas na raiz, no caule, no pedúnculo e nas folhas resulta na demanda nutricional da fração a ser restituída, enquanto o somatório das quantidades dos nutrientes acumuladas nos frutos e nas mudas resulta na demanda nutricional da fração a ser exportada. $\mathrm{O}$ somatório da demanda nutricional das frações a serem restituídas e exportadas resulta na demanda de nutrientes pela planta (Figura 1).
No FERTCALC-Abacaxi, adota-se uma dose de sustentabilidade, associada à manutenção de uma "reserva técnica", representada por uma "dose de segurança", na tentativa de evitar o esgotamento das reservas dos nutrientes no solo ao longo do tempo e garantir uma produtividade mínima de frutos em cultivos subsequentes (Cantarutti et al., 2007). Embora opcional, a utilização da dose de sustentabilidade no FERTCALC-Abacaxi deve ser sempre considerada, haja vista as quantidades elevadas de nutrientes exportadas nos frutos e nas mudas (Silva et al., 2006).

A dose de sustentabilidade do FERTCALC-Abacaxi consiste numa dose suplementar de nutrientes a ser acrescida à demanda de nutrientes pela planta e corresponde a todos os nutrientes, com exceção do N, a $60 \%$ da demanda nutricional da fração a ser exportada (frutos e mudas). Para $\mathrm{N}$, devido às perdas do nutriente no sistema solo-planta-atmosfera (lixiviação, imobilização, volatilização, etc.), a dose de sustentabilidade corresponde unicamente a $40 \%$ da demanda nutricional da fração a ser exportada. Somando-se a demanda de nutrientes pela planta com a demanda de sustentabilidade, obtém-se a demanda total pelo abacaxizeiro para determinado nutriente para alcançar a produtividade esperada.

O requerimento total de nutrientes pelo abacaxizeiro é então obtido dividindo-se o valor da demanda total pela taxa de recuperação pelo abacaxizeiro do nutriente aplicado via fertilizante $\left(\mathrm{TR}_{\mathrm{NF}}\right)$, uma vez que a planta não absorve $100 \%$ do nutriente aplicado, devido a fatores como perdas e competição com solo (Santos et al., 2008). A TR $\mathrm{TR}_{\mathrm{NF}}$ indica a eficiência da planta na absorção do nutriente proveniente do adubo e é definida como a quantidade 
Quadro 3. Valores de CUB nas diferentes partes dos abacaxizeiros Pérola e Smooth Cayenne, utilizados no FERTCALC-Abacaxi ${ }^{(1)}$

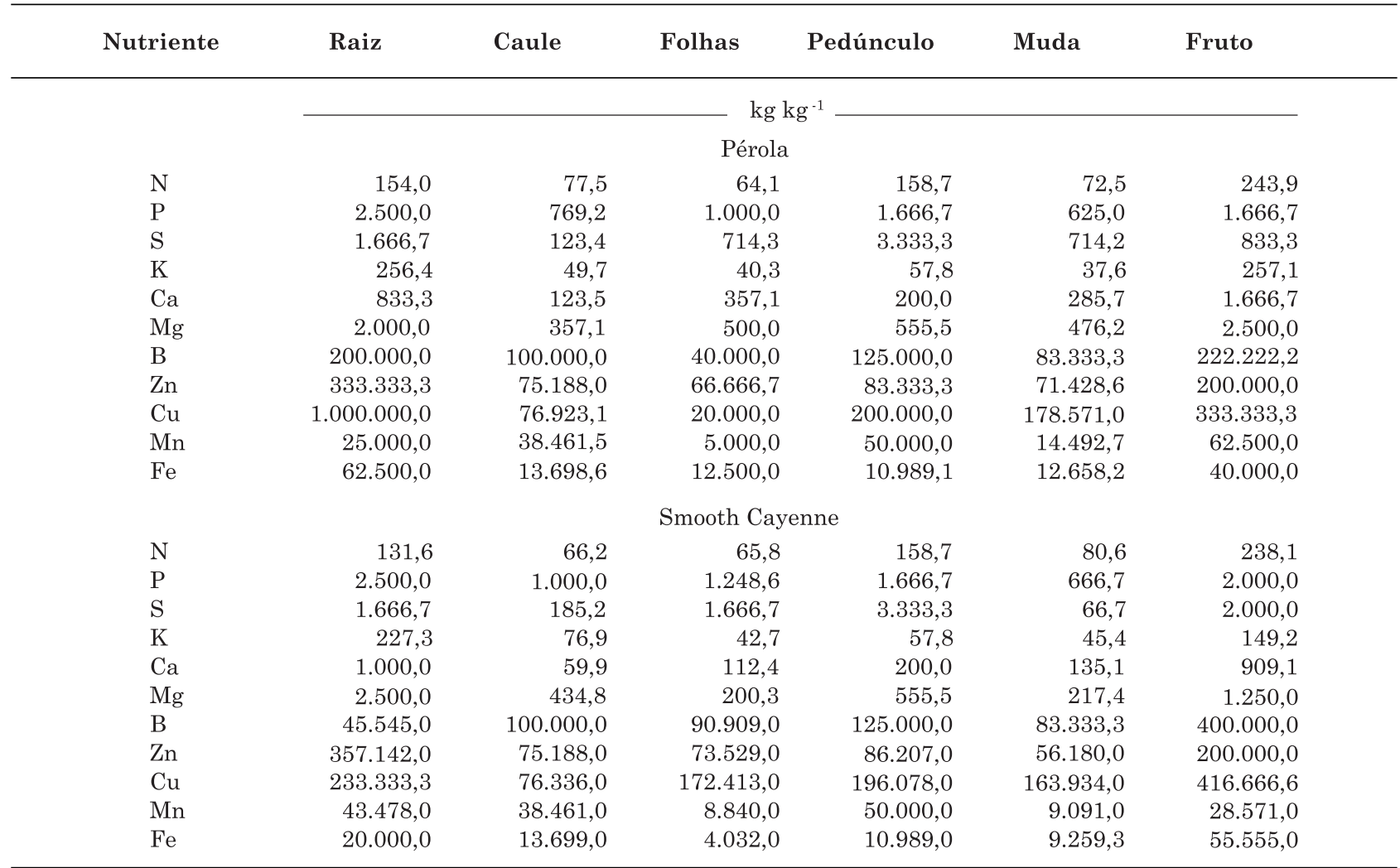

(1) Fonte: Adaptado de Silva (2006).

de nutriente absorvida por unidade de nutriente aplicada (Fageria, 1992).

Os valores da $\mathrm{TR}_{\mathrm{NF}}$ são influenciados por fatores edáficos ( $\mathrm{pH}$, teor e capacidade tampão, de nutrientes e matéria orgânica), climáticos (temperatura, radiação, precipitação), biológicos (micorrização), da própria planta (cultivar, idade e morfologia de raiz), e de manejo (dose, fonte e forma de aplicação do adubo), sendo a influência desses fatores, também, variável em função do nutriente considerado (Oliveira et al., 2005; Santos et al., 2008).

Como não foram encontrados dados que permitissem estimar o efeito dos diferentes fatores envolvidos nos valores das $\mathrm{TR}_{\mathrm{NF}}$ pelo abacaxizeiro, adotaram-se, na primeira versão do FERTCALC-Abacaxi, equações para N, P e K, em função da dose a ser aplicada, e para B e Zn em função da capacidade tampão (Quadro 4). Para Ca, Mg e S, foram utilizados valores médios extraídos do trabalho de Gadelha (1992) com o cultivar Pérola. Para nutrientes como $\mathrm{Cu}, \mathrm{Mn}$ e Fe, adotaramse taxas médias, considerando doses e fontes mais comuns dos nutrientes para culturas diversas.

\section{Subsistema suprimento}

O suprimento total de nutrientes estimado pelo FERTCALC-Abacaxi é representado pela seguinte expressão:

$$
\begin{gathered}
\mathrm{SUP}_{\text {total }}=\mathrm{SUP}_{\text {calagem }}+\mathrm{SUP}_{\mathrm{MOS}}+ \\
\mathrm{SUP}_{\text {solo }}+\mathrm{SUP}_{\text {res.org }}
\end{gathered}
$$

em que $\mathrm{SUP}_{\text {total }}$ : suprimento total de nutrientes,

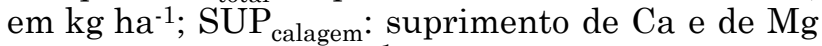
pela calagem, em kg ha-1; para os demais nutrientes o valor de $\mathrm{SUP}_{\text {calagem }}$ será igual a zero; $\mathrm{SUP}_{\mathrm{MOS}}$ : suprimento de nutrientes (apenas para N) pela matéria orgânica do solo, em kg ha ${ }^{-1}$; para os demais nutrientes o valor de $\mathrm{SUP}_{\mathrm{Mos}}$ será igual a zero; $\mathrm{SUP}_{\text {solo }}$ : suprimento de nutrientes pelo solo, em $\mathrm{kg} \mathrm{ha}{ }^{-1}$; para $\mathrm{N}$ o valor de $\mathrm{SUP}_{\text {solo }}$ será igual a zero; $\mathrm{SUP}_{\text {res.org: }}$ suprimento de nutrientes (todos) pelos resíduos orgânicos, em $\mathrm{kg} \mathrm{ha} \mathrm{ha}^{-1}$

\section{Suprimento de nutrientes (Ca e Mg) pela calagem}

No FERTCALC-Abacaxi, a necessidade de calagem (NC) pode ser determinada por dois métodos: (a) neutralização do $\mathrm{Al}^{3+}$ e elevação dos teores de $\mathrm{Ca}^{2+} \mathrm{e}$ de $\mathrm{Mg}^{2+}$; e (b) saturação por bases (Alvarez V. \& Ribeiro, 1999).

No primeiro método, são consideradas características do solo, a exemplo da capacidade tampão do solo (Y), bem como a tolerância da cultura à acidez trocável $\left(m_{t}\right)$ e as exigências da cultura em termos de Ca e de $\mathrm{Mg}(\mathrm{X})$. Para o abacaxizeiro, o valor de $\mathrm{m}_{\mathrm{t}}$ adotado é 
Quadro 4. Taxas de recuperação $\left(\mathrm{kg} \mathrm{kg}^{-1}\right)$ dos nutrientes aplicados via fertilizantes $\left(\mathrm{TR}_{\mathrm{NF}}\right)$ adotadas no FERTCALC-Abacaxi

\begin{tabular}{|c|c|c|}
\hline Nutriente & Equação/taxa ${ }^{(1)}$ & $\mathbf{R}^{2}$ \\
\hline Nitrogênio & $\begin{array}{l}\forall \mathrm{D}_{\mathrm{N}}<300, \mathrm{TR}_{\mathrm{NF}}(\mathrm{N})=0,68 \\
\mathrm{TR}_{\mathrm{NF}}(\mathrm{N})=0,8686-0,0006^{*} \mathrm{D}_{\mathrm{N}}, \forall 300 \leq \mathrm{D}_{\mathrm{N}} \leq 840 \\
\forall \mathrm{D}_{\mathrm{N}}>840, \mathrm{TR}_{\mathrm{NF}}(\mathrm{N})=0,36\end{array}$ & $\begin{array}{c}- \\
0,43 \\
-\end{array}$ \\
\hline Fósforo & $\begin{array}{l}\forall \mathrm{D}_{\mathrm{P}}<80, \mathrm{TR}_{\mathrm{NF}}(\mathrm{P})=0,42 \\
\mathrm{TR}_{\mathrm{NF}}(\mathrm{P})=45,0-0,1218^{*} \mathrm{D}_{\mathrm{P},} \forall 80 \leq \mathrm{D}_{\mathrm{P}} \leq 320 \\
\forall \mathrm{D}_{\mathrm{P}}>320, \mathrm{TR}_{\mathrm{NF}}(\mathrm{P})=0,10\end{array}$ & 0,84 \\
\hline Enxofre & $\mathrm{TR}_{\mathrm{NF}}(\mathrm{S})=0,59$ & - \\
\hline Potássio & $\begin{array}{l}\forall \mathrm{D}_{\mathrm{K}}<180, \mathrm{TR}_{\mathrm{NF}}(\mathrm{K})=0,70 \\
\mathrm{TR}_{\mathrm{NF}}(\mathrm{K})=75,67-0,0317^{*} \mathrm{D}_{\mathrm{K}}, \forall 180 \leq \mathrm{D}_{\mathrm{K}} \leq 857 \\
\forall \mathrm{D}_{\mathrm{K}}>857, \mathrm{TR}_{\mathrm{NF}}(\mathrm{K})=0,48\end{array}$ & $\overline{0}, 86$ \\
\hline Cálcio & $\mathrm{TR}_{\mathrm{NF}}(\mathrm{Ca})=0,60$ & - \\
\hline Magnésio & $\mathrm{TR}_{\mathrm{NF}}(\mathrm{Mg})=0,52$ & - \\
\hline Boro & $\mathrm{TR}_{\mathrm{NF}}(\mathrm{B})=5,282755 /\left(1-0,82839397 \mathrm{e}^{-0,34760374 \mathrm{DB}}\right)$ & 0,89 \\
\hline Zinco & $\operatorname{TR}_{\mathrm{NF}}(\mathrm{Zn})=4,11+0,0775^{*} \mathrm{P}_{\mathrm{rem}}$ & 0,93 \\
\hline Cobre & $\operatorname{TR}_{\mathrm{NF}}(\mathrm{Cu})=0,10$ & - \\
\hline Manganês & $\mathrm{TR}_{\mathrm{NF}}(\mathrm{Mn})=0,10$ & - \\
\hline Ferro & $\mathrm{TR}_{\mathrm{NF}}(\mathrm{Fe})=0,20$ & - \\
\hline
\end{tabular}

(1) Fonte: Adaptado de Silva (2006); $\mathrm{D}_{\mathrm{N}}, \mathrm{D}_{\mathrm{P}}, \mathrm{D}_{\mathrm{K}}$ e $\mathrm{D}_{\mathrm{B}}$ representam, respectivamente doses de $\mathrm{N}, \mathrm{P}, \mathrm{K}$ e $\mathrm{B}$ a serem aplicadas, e correspondem aos valores de demanda pela planta, em $\mathrm{kg} \mathrm{ha}^{-1}$; * significativo a $5 \%$.

de $15 \%$ e X é igual a $2,0 \mathrm{cmol}_{\mathrm{c}} \mathrm{dm}^{-3}$, sendo a NC obtida a partir da seguinte expressão:

$$
\mathrm{NC}=\mathrm{Y}\left[\mathrm{Al}^{3+}-\left(\mathrm{m}_{\mathrm{t}} \cdot \mathrm{t} / 100\right)\right]+\left[\mathrm{X}-\left[\left(\mathrm{Ca}^{2+}+\mathrm{Mg}^{2+}\right)\right]\right.
$$

em que NC: necessidade de calagem, em $\mathrm{kg} \mathrm{ha}^{-1}$; Y: valor relacionado à capacidade tampão do solo; $\mathrm{Al}^{3+}$ : acidez trocável, em $\mathrm{cmol}_{\mathrm{c}} \mathrm{dm}^{-3} ; \mathrm{m}_{\mathrm{t}}$ : saturação por $\mathrm{Al}^{3+}$ tolerada pela cultura, em \%; t: CTC efetiva, em $\mathrm{cmol}_{\mathrm{c}} \mathrm{dm}^{-3}$; e X: valor relacionado à exigência da cultura em Ca e $\mathrm{Mg}$, em $\mathrm{cmol}_{\mathrm{c}} \mathrm{dm}^{-3}$.

$\mathrm{O}$ valor de $\mathrm{Y}$ é variável com a capacidade tampão da acidez do solo, podendo ser estimado em função do $\mathrm{P}_{\text {rem }}$ (Alvarez V. \& Ribeiro, 1999).

$$
\begin{gathered}
\hat{\mathrm{Y}}=4,002-0,125901 \mathrm{P}_{\text {rem }}+0,001205\left(\mathrm{P}_{\mathrm{rem}}\right)^{2}- \\
0,00000362\left(\mathrm{P}_{\mathrm{rem}}\right)^{3} \\
\mathrm{R}^{2}=0,999
\end{gathered}
$$

em que, $\mathrm{P}_{\text {rem }}$ é expresso em $\mathrm{mg} \mathrm{L}^{-1}$.

Quando não se dispõe do valor de $\mathrm{P}_{\text {rem }}$ do solo na análise, pode-se estimar esse valor em função do teor de argila, com base na equação obtida por Freire (2001):

$$
\begin{gathered}
P_{\text {rem }}=52,44-0,9646 * * \operatorname{Arg}+0,005^{* *} \mathrm{Arg}^{2} \\
R^{2}=0,75
\end{gathered}
$$

em que $\mathrm{P}_{\text {rem }}$ é expresso em $\mathrm{mg} \mathrm{L}^{-1}$ e Arg é o teor de argila em \%.

No método da saturação por bases, procura-se elevar o valor de saturação por bases atual do solo $\left(\mathrm{V}_{\mathrm{a}}\right)$ para um valor esperado $\left(\mathrm{V}_{\mathrm{e}}\right)$, corrigindo-se, assim, o $\mathrm{pH}$ para um valor considerado adequado para a cultura. Para o abacaxizeiro, utiliza-se o valor de $50 \%$ para $\mathrm{V}_{\mathrm{e}}$ e procura-se manter o teor de $\mathrm{Mg}^{2+}$ acima de 0,6 $\mathrm{cmol}_{\mathrm{c}} \mathrm{dm}^{-3}$ (Souza, 1999).

$\mathrm{O}$ valor da NC por este método é determinado pela seguinte expressão:

$$
\mathrm{NC}=\mathrm{T}\left(\mathrm{V}_{\mathrm{e}}-\mathrm{V}_{\mathrm{a}}\right) / 100
$$

em que NC: necessidade de calagem, em t ha-1; $\mathrm{T}$ : $\mathrm{CTC}$ a pH 7: $\mathrm{SB}+(\mathrm{H}+\mathrm{Al})$, em $\mathrm{cmol}_{\mathrm{c}} \mathrm{dm}^{-3}$; SB: soma de bases: $\mathrm{Ca}^{2+}+\mathrm{Mg}^{2+}+\mathrm{K}^{+}+\mathrm{Na}^{+}$, em $\mathrm{cmol}_{\mathrm{c}} \mathrm{dm}^{-3} ; \mathrm{V}_{\mathrm{a}}$ : saturação por bases atual do solo: $100 \mathrm{SB} / \mathrm{T}$, em \%; $\mathrm{V}_{\mathrm{e}}$ : saturação por bases esperada para a cultura.

\section{Suprimento pela matéria orgânica do solo}

No FERTCALC-Abacaxi, a mineralização da matéria orgânica do solo serve apenas como fonte de N. Assim, para estimar o suprimento de $\mathrm{N}$ pela matéria orgânica do solo, adota-se a equação de cinética de mineralização da matéria orgânica para solos brasileiros (Parentoni et al., 1988; Vasconcelos et al., 1999):

$$
\begin{gathered}
\operatorname{SUP}(\mathrm{N})_{\text {solo }}=\left(\mathrm{M}_{\text {solo }} \times \mathrm{N}_{\text {total }} \times 1,48 / 100\right) e^{0,006 \mathrm{t}} \\
\mathrm{M}_{\text {solo }}=1.000 \times 1.000 \times \mathrm{PER} \times \text { d.s. } \mathrm{e} \\
\mathrm{N}_{\text {total }}=\left[\mathrm{T}_{\mathrm{MOS}}(58 / 100) / 2\right] / 1.000
\end{gathered}
$$

em que $\operatorname{SUP}(\mathrm{N})_{\text {solo: }}$ suprimento de $\mathrm{N}$ pelo solo durante o ciclo do abacaxizero, em $\mathrm{kg} \mathrm{ha}{ }^{-1} ; \mathrm{M}_{\text {solo }}$ : massa de solo na camada delimitada pela profundidade efetiva do sistema radicular do abacaxizeiro, em 
$\mathrm{kg} \mathrm{ha}{ }^{-1} ; \mathrm{N}_{\text {total }}$ : nitrogênio total do solo, em kg kg-1 de solo; 1,48: fração do $\mathrm{N}$ potencialmente mineralizável a partir do $\mathrm{N}$ total, em \%; PER: profundidade do sistema radicular do abacaxizeiro, em dm; d.s.: densidade do solo, em kg dm${ }^{-3}$; $\mathrm{T}_{\mathrm{MOs}}$ : teor de matéria orgânica do solo, em dag kg-1; e t: tempo de absorção de $\mathrm{N}$ pela cultura, em dias (210).

\section{Suprimento de nutrientes pelo solo}

A quantidade de nutrientes suprida pelo solo $\left(\mathrm{mg} \mathrm{dm} \mathrm{dm}^{-3}\right)$ é obtida dividindo-se o teor do nutriente encontrado na análise de solo pela taxa de recuperação do extrator do respectivo nutriente aplicado ao solo, considerando a profundidade efetiva do sistema radicular do abacaxizeiro de 0,20 $\mathrm{m}$ (Inforzatto et al., 1968). O Sistema contempla os seguintes extratores utilizados para análise química: Mehlich-1 (P, K, Fe, $\mathrm{Mn}$ e Zn), Resina (P e K), $\mathrm{KCl}$ (Ca e Mg), DTPA (Cu, $\mathrm{Fe}, \mathrm{Mn}$ e $\mathrm{Zn}), \mathrm{CaCl}_{2}$ e água quente (B) e $\mathrm{Ca}\left(\mathrm{H}_{2} \mathrm{PO}_{4}\right)$ em HOAc $(\mathrm{S})$. Em relação às taxas de recuperação pelos extratores, o Sistema considera o efeito da capacidade tampão do solo para $\mathrm{P}, \mathrm{S}$ e $\mathrm{Zn}$ extraídos por Mehlich-1, além de K, B e P pela Resina. Para Ca e Mg são consideradas taxas fixas, enquanto, para micronutrientes, as taxas se relacionam com os valores de P-rem, teores de argila e de matéria orgânica (Quadro 5).

\section{Suprimento de nutrientes pelos resíduos orgânicos}

O suprimento de nutrientes provenientes dos resíduos orgânicos do abacaxizeiro é contabilizado no balanço nutricional a partir do segundo ciclo, utilizando-se informações sobre a quantidade de resíduos produzida no ciclo anterior, os teores de nutrientes na matéria seca dos resíduos e a taxa de mineralização desses nutrientes nos resíduos (Quadro 6). A quantidade de resíduos produzida no ciclo anterior é estimada substituindo-se a produtividade obtida no primeiro ciclo nas equações que relacionam produtividade e matéria seca (Quadro 2), multiplicando-se o valor obtido pelo fator 0,95, visando descontar a contribuição das mudas. Em seguida, multiplicando-se a quantidade de matéria seca dos resíduos pelos respectivos teores de nutrientes e depois pelo valor da taxa de mineralização dos resíduos orgânicos, determina-se o suprimento de nutrientes pelos resíduos orgânicos.

Estabelecidos os valores de requerimento e de suprimento de nutrientes, subsituem-se seus valores na equação do balanço nutricional (Equação 9). Caso o balanço seja positivo (REQ > SUP), recomenda-se a aplicação de fertilizantes; caso seja negativo ou nulo (REQ $\leq \mathrm{SUP})$, não se recomenda a aplicação de fertilizantes:

$$
\mathrm{BN}=\mathrm{REQ}_{\text {total }}-\mathrm{SUP}_{\text {total }}
$$

em que BN: balanço nutricional para o nutriente " $\mathrm{X}$ ", em kg ha-1; $\mathrm{REQ}_{\text {total }}$ : requerimento total do nutriente "X" para a produtividade a ser efetivamente alcançada e dose de sustentabilidade, em $\mathrm{kg} \mathrm{ha}^{-1}$; $\mathrm{SUP}_{\text {total }}$ : suprimento total do nutriente "X", em $\mathrm{kg} \mathrm{ha} \mathrm{h}^{-1}$.

Quadro 5. Taxas de recuperação pelo extrator do nutriente aplicado ao solo (TR), em $\mathrm{mg} \mathrm{dm}^{-3}$, variável ou não, com o extrator, teor de argila (Arg) em \%, teor de matéria orgânica (MO) em dag kg-1, e valores de $P_{\text {rem }}$ em mg L $\mathbf{~}^{-1}$

\begin{tabular}{|c|c|c|c|}
\hline Nutriente & Extrator & Equação & $\mathbf{R}^{2}$ \\
\hline Fósforo $^{(1)}$ & $\begin{array}{l}\text { Mehlich-1 } \\
\text { Resina }\end{array}$ & $\begin{array}{l}\mathrm{TR}_{\mathrm{P}}=0,0672821+0,012165^{* *} \text { P-rem } \\
\mathrm{TR}_{\mathrm{P}}=0,419^{* * *} \mathrm{P}_{\text {rem }}{ }^{0,128099}\end{array}$ & $\begin{array}{l}0,68 \\
0,69\end{array}$ \\
\hline Potássio ${ }^{(1)}$ & $\begin{array}{l}\text { Mehlich-1 } \\
\text { Resina }\end{array}$ & $\begin{array}{l}\mathrm{TR}_{\mathrm{K}}=0,6555+0,0068^{* *} \text { P-rem }, \forall 1 \leq \mathrm{P}-\mathrm{rem} \leq 40 \\
\mathrm{TR}_{\mathrm{K}}=0,6619+0,014355^{*} \text { P-rem }-0,00364^{\circ} \text { P-rem }{ }^{2}\end{array}$ & $\begin{array}{l}0,74 \\
0,75\end{array}$ \\
\hline Cálcio ${ }^{(1)}$ & $\mathrm{KCl} 1 \mathrm{~mol} \mathrm{~L}^{-1}$ & $\mathrm{TR}_{\mathrm{Ca}}=0,7661$ & - \\
\hline Magnésio ${ }^{(1)}$ & $\mathrm{KCl} 1 \mathrm{~mol} \mathrm{~L}^{-1}$ & $\mathrm{TR}_{\mathrm{Mg}}=0,7990$ & - \\
\hline Enxofre $^{(1)}$ & $\mathrm{Ca}\left(\mathrm{H}_{2} \mathrm{PO}_{4}\right)+\mathrm{HOAc}$ & $\mathrm{TR}_{\mathrm{S}}=0,04+0,0057^{* *} \mathrm{P}-\mathrm{rem}$ & 0,95 \\
\hline Boro $^{(1)}$ & $\begin{array}{l}\text { Água quente } \\
\mathrm{CaCl}_{2}\end{array}$ & $\begin{array}{l}\mathrm{TR}_{\mathrm{B}}=0,1726+0,02693 \mathrm{P}-\mathrm{rem}{ }^{0,5}+0,00696188^{*} \mathrm{P}-\mathrm{rem}-0,0007568^{*} \mathrm{P}-\mathrm{rem}^{1,5} \\
\mathrm{TR}_{\mathrm{B}}=0,49-0,0441^{* * *} \mathrm{MO}+0,0045^{*} \mathrm{P}-\mathrm{rem}\end{array}$ & $\begin{array}{l}0,63 \\
0,61\end{array}$ \\
\hline Cobre $^{(2)}$ & $\begin{array}{l}\text { Mehlich-1 } \\
\text { DTPA }\end{array}$ & $\begin{array}{l}\mathrm{TR}_{\mathrm{Cu}}=1,05-0,0046^{* *} \mathrm{Arg}-0,0851^{* * *} \mathrm{MO} \\
\mathrm{TR}_{\mathrm{Cu}}=0,81-0,0058^{* * *} \mathrm{Arg}-0,0347^{*} \mathrm{MO}\end{array}$ & $\begin{array}{l}0,84 \\
0,76\end{array}$ \\
\hline Ferro $^{(3)}$ & $\begin{array}{l}\text { Mehlich-1 } \\
\text { DTPA }\end{array}$ & $\begin{array}{l}\mathrm{TR}_{\mathrm{Fe}}=0,409 \\
\mathrm{TR}_{\mathrm{Fe}}=0,192\end{array}$ & - \\
\hline Manganês ${ }^{(3)}$ & $\begin{array}{l}\text { Mehlich-1 } \\
\text { DTPA }\end{array}$ & $\begin{array}{l}\mathrm{TR}_{\mathrm{Mn}}=0,881 \\
\mathrm{TR}_{\mathrm{Mn}}=0,694\end{array}$ & - \\
\hline Zinco $^{(2)}$ & $\begin{array}{l}\text { Mehlich-1 } \\
\text { DTPA }\end{array}$ & $\begin{array}{l}\mathrm{TR}_{\mathrm{Zn}}=0,360254-0,002338 \text { P-rem }+0,0001198^{* *} \text { P-rem }{ }^{2} \\
\mathrm{TR}_{\mathrm{Zn}}=0,48-0,0040^{* *} \mathrm{Arg}\end{array}$ & $\begin{array}{l}0,93 \\
0,31\end{array}$ \\
\hline
\end{tabular}


Quadro 6. Teor e taxas de mineralização (TM) de nutrientes nos resíduos orgânicos adotados no FERTCALCAbacaxi

\begin{tabular}{|c|c|c|c|c|c|}
\hline Nutriente & Teor $^{(1)}$ & $\mathbf{T M}^{(2)}$ & Nutriente & $\operatorname{Teor}^{(1)}$ & $\mathbf{T M}^{(2)}$ \\
\hline & dag kg ${ }^{-1}$ & $\mathrm{~kg} \mathrm{~kg}-1^{-1}$ & & $\mathrm{mg} \mathrm{kg}^{-1}$ & $\mathrm{~kg} \mathrm{~kg}^{-1}$ \\
\hline $\mathrm{N}$ & 1,03 & $0,65 \pm 0,13$ & $\mathrm{~B}$ & 26,0 & $0,50 \pm 0,10$ \\
\hline $\mathrm{P}$ & 0,19 & $0,60 \pm 0,12$ & $\mathrm{Cu}$ & 10,0 & $0,25 \pm 0,10$ \\
\hline $\mathrm{K}$ & 1,94 & $0,85 \pm 0,17$ & $\mathrm{Fe}$ & 180,0 & $0,25 \pm 0,10$ \\
\hline $\mathrm{Ca}$ & 0,31 & $0,55 \pm 0,11$ & $\mathrm{Mn}$ & 270,0 & $0,25 \pm 0,10$ \\
\hline $\mathrm{Mg}$ & 0,19 & $0,55 \pm 0,11$ & $\mathrm{Zn}$ & 30,0 & $0,30 \pm 0,06$ \\
\hline $\mathrm{S}$ & 0,23 & $0,60 \pm 0,12$ & & & \\
\hline
\end{tabular}

${ }^{(1)}$ Fonte: Adaptado de Silva (2006). ${ }^{(2)}$ Oliveira et al. (2005).

\section{Aplicação do sistema}

Foram feitas simulações das recomendações de N, $\mathrm{P}$ e K pelo FERTCALC-Abacaxi para os cultivares Pérola e Smooth Cayenne, considerando variações dos fatores peso do fruto, sistema e densidade de plantio. Nas simulações, utilizou-se um solo de baixa fertilidade, com as seguintes características: $\mathrm{pH}=5,1$; $4 \mathrm{mg} \mathrm{dm}^{-3}$ de $\mathrm{P}$ (Mehlich-1); $42 \mathrm{mg} \mathrm{dm}^{-3}$ de K; 2,5 dag kg-1 de matéria orgânica; P-rem $=30 \mathrm{mg} \mathrm{L}^{-1}$ e $22 \%$ de argila. Para ambos os cultivares e sistema de plantio, adotaram-se as densidades teóricas do quadro 1, corrigidas pelo fator de nível tecnológico médio $(0,8)$, adotando-se a variação do peso dos frutos entre 0,8 e 2,0 kg para o cultivar Pérola e entre 0,9 e $2,5 \mathrm{~kg}$ para o cultivar Smooth Cayenne.

Realizaram-se ainda comparações entre as doses recomendadas pelo FERTCALC-Abacaxi e pelas tabelas de adubação dos Estados da Paraíba (EMATERPB) (EMATER, 1979) e da Bahia (EMATER-BA) (Souza, 1999) para o cultivar Pérola, o mais plantado no Brasil. Nessas comparações, foram utilizados solos de fertilidade baixa (3,8 $\mathrm{mg} \mathrm{dm}^{-3}$ de P-Mehlich-1; Prem $=33,1 \mathrm{mg} \mathrm{L}^{-1} ; 21 \mathrm{mg} \mathrm{dm}^{-3}$ de $\mathrm{K} ; 1,0 \mathrm{dag} \mathrm{kg}^{-1} \mathrm{de}$ matéria orgânica) e alta (16,1 $\mathrm{mg} \mathrm{dm}^{-3} \mathrm{de}$ P-Mehlich-1; P-rem = 41,9 $\mathrm{mg} \mathrm{L}^{-1} ; 200 \mathrm{mg} \mathrm{dm}^{-3}$ de K; 3,05 dag kg-1 de matéria orgânica), considerando-se faixas de produtividades esperadas entre 27 e $67 \mathrm{t} \mathrm{ha}^{-1}$ e produtividade de $40 \mathrm{t} \mathrm{ha}^{-1}$ no primeiro cultivo.

\section{RESULTADOS E DISCUSSÃO}

As simulações realizadas mostraram, independente do cultivar e do sistema de plantio (fileiras simples ou duplas), que as doses recomendadas pelo FERTCALCAbacaxi aumentaram com a elevação do peso do fruto e da densidade de plantio (Figura 2), o que se deve ao aumento da demanda nutricional por cultivos mais adensados (Zang \& Bartholomew, 1993). Para frutos mais pesados (Spironello et al., 2004), não há critérios previstos nas tabelas (Souza, 1999; Silva, 2006). No entanto, a relação entre os fatores densidade, peso do fruto e demanda precisa ser melhor estudada, no sentido de esclarecer os efeitos na eficiência de absorção de nutrientes pela cultura e consequentemente na definição das doses.

Comparando-se as doses recomendadas pelo FERTCALC-Abacaxi para o cultivar Pérola com as das tabelas dos Estados da Paraíba (EMATER-PB) e da Bahia (EMATER-BA) (Figura 3), verifica-se que, ao contrário das tabelas que estabelecem doses fixas dos nutrientes, definidas em função do teor de nutriente no solo, mas independentes da produtividade esperada, as doses recomendadas pelo FERTCALC-Abacaxi variaram de forma contínua em função da produtividade esperada, do teor e da capacidade tampão de nutrientes no solo e dos cultivos.

Para N, o FERTCALC-Abacaxi recomenda maiores doses em relação às tabelas tanto para solo com teor de matéria orgânica baixo, quanto para alto no primeiro cultivo. No segundo cultivo, as doses também são maiores em relação à EMATER-BA, independente do teor de matéria orgânica, porém, em relação à EMATER-PB, as doses são maiores apenas para produtividades $>35 \mathrm{t} \mathrm{ha}^{-1}$ no solo com teor de matéria orgânica baixo e $>45 \mathrm{t} \mathrm{ha}^{-1}$ no solo com teor de matéria orgânica alto (Figura 3). As menores doses de N no solo de fertilidade alta e a redução nas doses no segundo cultivo, em ambos os solos, se devem à contribuição da mineralização da matéria orgânica e à contabilização no balanço nutricional do suprimento de nutrientes dos resíduos orgânicos do cultivo anterior, respectivamente.

Quanto ao P, o FERTCALC-Abacaxi recomenda maiores doses para solo com teor de $\mathrm{P}$ baixo e alta capacidade tampão $\left(\mathrm{P}\right.$-rem $\left.=33,1 \mathrm{mg} \mathrm{L}^{-1}\right)$ no primeiro cultivo em toda a faixa de produtividade, e no solo com teor de $\mathrm{P}$ alto e baixa capacidade tampão (P-rem $=41,9 \mathrm{mg} \mathrm{L}^{-1}$ ) para produtividades $>45 \mathrm{t} \mathrm{ha}^{-1} \mathrm{em}$ relação à EMATER-BA e $>55 \mathrm{t}^{\text {ha }}{ }^{-1}$ em relação à EMATER-PB (Figura 3). No segundo cultivo, entretanto, o Sistema recomenda menores doses em relação à EMATER-PB para produtividades até $50 \mathrm{t} \mathrm{ha}^{-1}$ no solo com teor de $\mathrm{P}$ baixo e alta capacidade 

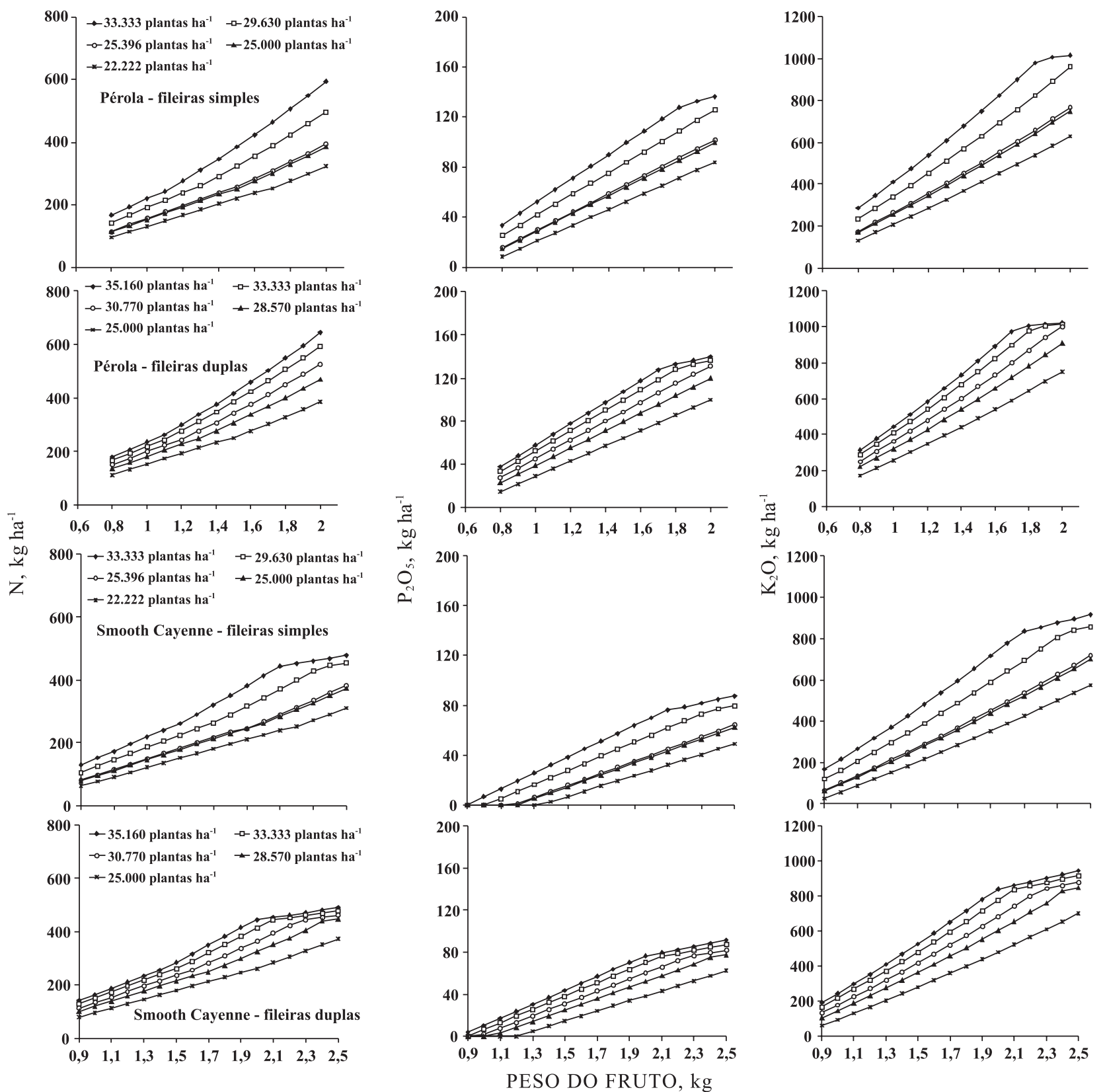

Figura 2. Doses de N, P e K recomendadas pelo FERTCALC-Abacaxi para os cultivares Pérola e Smooth Cayenne, em função do peso do fruto e da densidade de plantio, nos sistemas de fileiras simples e duplas.

tampão e até $30 \mathrm{t} \mathrm{ha}^{-1}$ em relação à EMATER-PB (Figura 2). No solo com teor de P alto e baixa capacidade tampão, o Sistema recomenda doses de P para produtividades a partir de $50 \mathrm{t} \mathrm{ha}^{-1}$, sendo, em geral, inferiores em relação às tabelas. Tais resultados demonstram que a pequena demanda de $\mathrm{P}$ pelo abacaxizeiro foi em parte assegurada pelo suprimento pela fração mineral do solo e pelos resíduos orgânicos do cultivo anterior, concordando assim com as afirmações de Marchal (1971) sobre a alta capacidade de utilização de $\mathrm{P}$ pelo abacaxizeiro. Contudo, esse é um ponto que precisa ser melhor explorado em futuras versões do Sistema (Souza, 1999; Silva, 2006).
Para K, as doses recomendadas pelo FERTCALCAbacaxi foram, em geral, elevadas, e maiores em relação às tabelas em toda a faixa de produtividade tanto no primeiro quanto no segundo cultivo em solo com teor de K baixo e para o primeiro cultivo no solo com teor de K alto (Figura 3). Contudo, no segundo cultivo, em solo com teor de K alto, o FERTCALCAbacaxi só recomenda doses de K para produtividade $>30 \mathrm{t} \mathrm{ha}^{-1}$, sendo inferiores em relação às tabelas até produtividade de 35 (EMATER-BA) e $43 \mathrm{t} \mathrm{ha}^{-1}$ (EMATER-PB) (Figura 3).

As elevadas doses de $\mathrm{K}$ recomendadas pelo FERTCALC-Abacaxi podem ser explicadas por um 

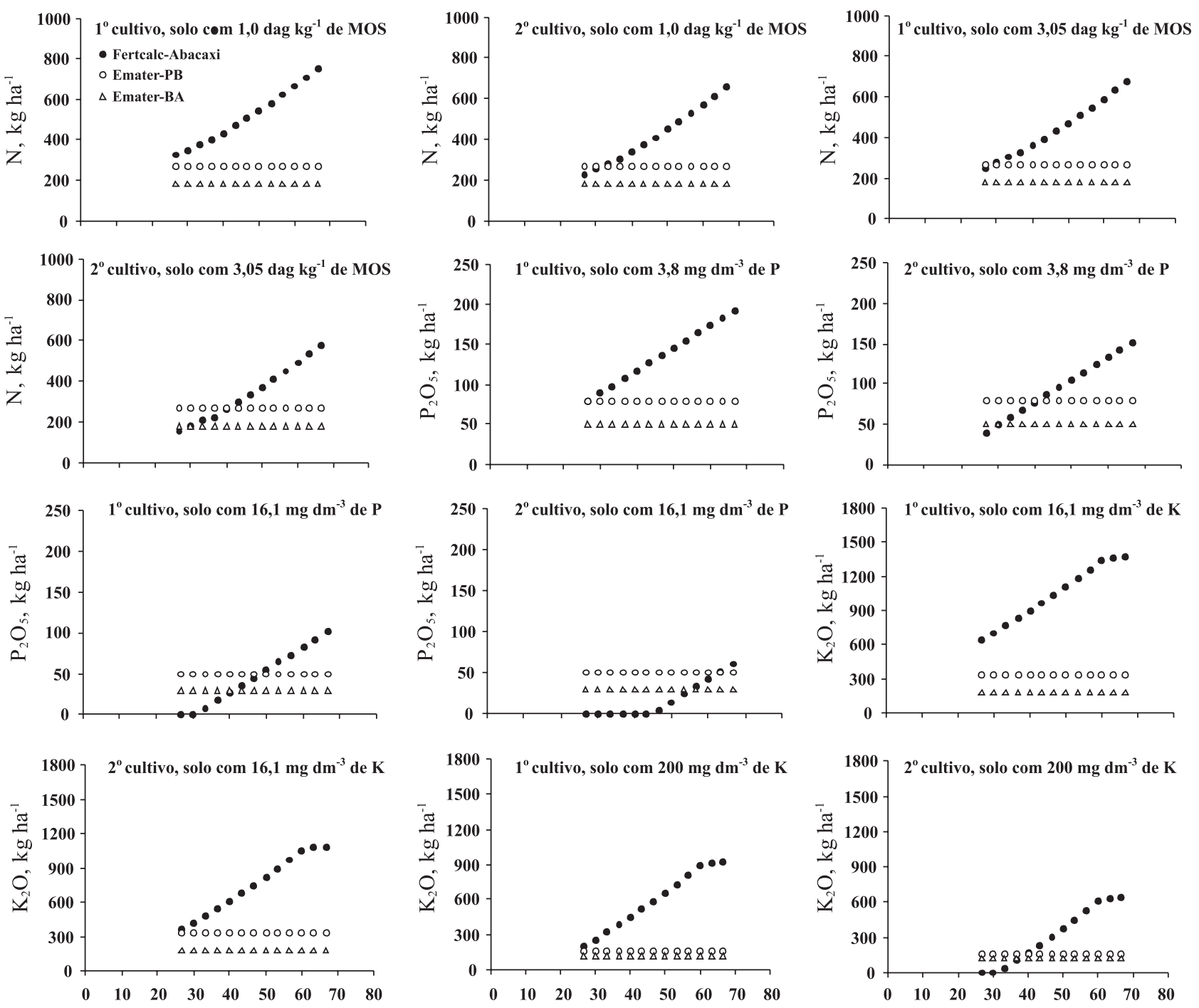

Figura 3. Doses de $\mathrm{N}, \mathrm{P}_{2} \mathrm{O}_{5}$ e $\mathrm{K}_{2} \mathrm{O}$ recomendadas pelo FERTCALC-Abacaxi e pelas tabelas dos Estados da Paraíba (EMATER-PB) e Bahia (EMATER-BA) para o cultivar Pérola, em função da produtividade esperada e dos teores de nutrientes no solo, no primeiro e segundo cultivos.

provável "consumo de luxo" pela planta, o qual estaria superestimando os teores e elevando a demanda de $\mathrm{K}$ pela cultura. Além disso, a não contabilização no balanço nutricional do suprimento de $\mathrm{K}$ a partir de formas não trocáveis e a possível subestimação dos valores da $\mathrm{TR}_{\mathrm{NF}}$ estaria contribuindo para aumentar as doses (Silva, 2006). Futuros trabalhos precisam esclarecer estas questões no sentido de melhorar a parametrização do Sistema e a qualidade das recomendações. Por outro lado, a redução nas doses recomendadas pelo FERTCALC-Abacaxi no segundo cultivo se deve à contribuição dos resíduos orgânicos do cultivo anterior. De acordo com Malézieux \& Bartholomew (2003), a quantidade de resíduos orgânicos produzida pelo abacaxizeiro é bastante elevada (entre 40 e 60 t ha $^{-1}$ de matéria seca), podendo restituir ao solo até $200 \mathrm{~kg} \mathrm{ha}^{-1} \mathrm{de} \mathrm{K}$, dependendo da forma como são manejados.

\section{CONCLUSÕES}

1. As simulações realizadas com o FERTCALCAbacaxi mostraram que o Sistema recomenda maiores doses de N, P e K para o cultivar Pérola, para plantios mais adensados e para obtenção de frutos mais pesados e que, ao contrário das tabelas, elas variam de forma contínua com a produtividade esperada e com o teor de nutrientes no solo, sendo maiores no primeiro em relação ao segundo ciclo.

2. Por contemplar na sua estrutura maior número de fatores que influenciam na resposta da cultura à adubação (produtividade esperada, cultivar, peso do fruto, sistema e densidade de plantio), além de considerar a contribuição dos nutrientes provenientes dos residuos orgânicos, o FERTCALC-Abacaxi pode ser considerado uma alternativa mais versátil para o 
estabelecimento de doses de fertilizantes e corretivos para o abacaxizeiro. Contudo, o Sistema precisa ser aperfeiçoado e ter seu desempenho avaliado em condições práticas.

\section{AGRADECIMENTOS}

À CAPES e ao CNPq pela concessão de bolsas ao primeiro e segundo autores, respectivamente; ao BNB pelo apoio financeiro; ao CCA/UFPB e à EMEPA-PB pelo suporte técnico e logístico.

\section{LITERATURA CITADA}

ALMEIDA, C.O.; VILAR, L.C.; SOUZA, L.F.S.; REINHARDT, D.H. \& MACEDO, M.C. Peso do abacaxi: Um termo em discussão. Bahia Agríc., 6:1-6, 2005.

ALVAREZ V., V.H. \& RIBEIRO, A.C. Calagem In: RIBEIRO, A.C.; GUIMARÃES, P.T.G. \& ALVAREZ V., V.H., eds. Recomendação para o uso de corretivos e fertilizantes em Minas Gerais. $5^{\text {a }}$ aproximação. Viçosa, MG, CFSEMG, 1999. p.43-60.

ASPIAZÚ, I. Extração de ferro e manganês por Mehlich-1, Mehlich-3 e DTPA em solos de Minas Gerais e da Bahia. Viçosa, MG, Universidade Federal de Viçosa, 2004. 49p. (Tese de Mestrado).

BARROS, N.F.; NOVAIS, R.F.; TEIXEIRA, J.L. \& FERNANDES FILHO, E.I. NUTRICALC 2.0 - Sistema para cálculo del balance nutricional y recomendación de fertilizantes para el cultivo de eucalipto. Bosque, 16:129-131, 1995.

CANTARUTTI, R.B.; BARROS, N.F.; MARTINEZ, H.E.P. \& NOVAIS, R.F. Avaliação da fertiliddae do solo e recomendação de fertilizantes. In: NOVAIS, R.F.; ALVAREZ V., V.H.; BARROS, N.F.; FONTES, R.L.F.F.; CANTARUTTI, R.B. \& NEVES, J.C.L., eds. Fertilidade do solo. Viçosa, MG, Sociedade Brasileira de Ciência do Solo, 2007. p.645-737.

EMPRESA DE ASSISTÊNCIA TÉCNICA E EXTENSÃO RURAL DA PARAÍBA - EMATER-PB. Sugestões de adubação para o Estado da Paraíba: 1a Aploximação. João Pessoa, 1979. 105p.

FAGERIA, N.K. Maximizing crop yields. New York, Marcel Dekker, 1992. 274p.

FREIRE, F.J. Sistema para cálculo do balanço nutricional e recomendação de corretivos e fertilizantes para cana-deaçúcar. Viçosa, MG, Universidade Federal de Viçosa, 2001. 87p. (Tese de Doutorado).

GADELHA, C.A.A. Utilização da mistura calcário e gesso na cultura do abacaxi em solos de Tabuleiros Costeiros Paraibanos. Areia, Universidade Federal da Paraíba, 1992. 95p. (Trabalho de Graduação)

HAEFELE, S.M.; WOPEREIS, M.C.S.; NDIAYE, M.K.; BARRO, S.E. \& OULDISSELMOU, M. Internal nutrient efficiencies, fertilizer recovery rates and indigenous nutrient supply of irrigated lowland rice in Sahelian West Africa. Field Crops Res., 80:19-32, 2003.
INFORZATO, R.; GIACOMELLI, E.J. \& ROCHELLE, L.A. Sistema radicular do abacaxizeiro aos 4,8 e 12 meses, plantado no início da estação seca, em solo Latosol Vermelho Escuro-Orto. Bragantia, 27:135-141, 1968.

MALÉZIEUX, E. \& BARTHOLOMEW, D.P. Plant nutrition. In: BARTHOLOMEW, D.P.; PAUL, R.E. \& ROHRBACH, K.G., eds. The Pineapple: Botany, production and uses. Honolulu, CAB, 2003. p.143-165.

MARCHAL, J. Le phosphore chez l'ananas. Fruits, 26:189205, 1971.

OLIVEIRA, F.H.T.; NOVAIS, R.F.; ALVAREZ V., V.H. \& CANTARUTTI, R.B. Desenvolvimento de um sistema para recomendação de adubação para a cultura da bananeira. R. Bras. Ci. Solo, 29:131-143, 2005.

PARENTONI, S.N.; FRANÇA, G.E. \& BAHIA FILHO, A.F.C. Avaliação dos conceitos de quantidade e intensidade de mineralização de nitrogênio para trinta solos do Rio Grande do Sul. R. Bras. Ci. Solo, 12:225-229, 1988.

POSSAMAI, J.M. Sistema de recomendação de corretivos e fertilizantes para o cultivo do algodoeiro. Viçosa, MG, Universidade Federal de Viçosa, 2003. 91p. (Tese de Mestrado)

SANTOS NETO, J.A. Taxas de recuperação de zinco, cobre e boro por diferentes extratores em solos da Bahia e de Minas Gerais. Viçosa, MG, Universidade Federal de Viçosa, 2003. 51p.(Tese de Mestrado)

SANTOS, F.C.; NEVES, J.C.L.; NOVAIS, R.F.; ALVAREZ V., V.H. \& SEDIYAMA, C.S. Modelagem da recomendação de fertilizantes para a cultura da soja. R. Bras. Ci. Solo, 32:1661-1674, 2008.

SILVA, A.P. Sistema para recomendação de fertilizantes e corretivos para a cultura do abacaxizeiro. Viçosa, MG, Universidade Federal de Viçosa, 2006. 181p. (Tese de Doutorado)

SOUZA, L.F.S. Exigências edáficas e nutricionais. In: CUNHA, G.A.P.; CABRAL, J.R.S. \& SOUSA, L.F.S. O abacaxizeiro: Cultivo, agroindústria e economia. Brasília, Embrapa, 1999. p.67-83.

SPIRONELLO, A.; QUAGGIO, J.A.; TEIXEIRA, L.A.J.; FURLANI, P.R. \& SIGRIST, J.M.M. Pineapple yield and fruit quality affected by NPK fertilization in a tropical soil. R. Bras. Frutic., 26:155-159, 2004.

TEIXEIRA, L.A.J.; SPIRONELLO, A.; FURLANI, P.R. \& SIGRIST, J.M.M. Parcelamento da adubação NPK em abacaxizeiro. R. Bras. Frutic., 24:219-224, 2002.

TOMÉ JÚNIOR, J.B. \& NOVAIS, R.F. Utilização de modelos como alternativa às tabelas de recomendação de adubação. B. Inf. SBCS, 25:1-8, 2000.

VASCONCELLOS, C.A.; MARRIEL, I.E.; SANTOS, F.G. \& MAGALHÃES, C.A.O. Influência do manejo de solo e do resíduo de sorgo na mineralização do nitrogênio e a relação N-NH${ }_{4}^{+}$e N-NO ${ }_{3}^{-}$. In: ENCONTRO BRASILEIRO SOBRE SUBSTÂNCIAS HÚMICAS, 3., Santa Maria, 1999. Anais. Santa Maria, IHSS, 1999. p.189-199.

WITT, C.; DOBERMANN, S.; ABDULRACHMAN, S. \& GINES, H.C. Internal nutrients eficiences of irrigated lowland rice in tropical and subtropical Asia. Field Crops Res., 63:113-138, 1999.

ZHANG, J. \& BARTHOLOMEW, D.P. Effect of plant population density on growth and dry matter partitioning of pineapple. Acta Hortic., 425:363-376, 1993. 